\title{
Photodynamics of Asymmetric Di-Iron-Cyano Hydrogenases Examined by Time-Resolved Mid-Infrared Spectroscopy
}

\author{
Amber Meyers, ${ }^{1}$ Edwin J. Heilweil ${ }^{2}$ and Christopher J. Stromberg ${ }^{1}$ \\ ${ }^{1}$ Department of Chemistry and Physics, Hood College, Frederick, MD 21701-8524, USA \\ ${ }^{2}$ Nanoscale Device Characterization Division, Physical Measurement Laboratory, National Institute of Standards \\ and Technology (NIST), Gaithersburg, MD 20899, USA
}

\section{Supplemental Information}

\section{Contents:}

Table S1. Symmetry assignments for ground state isomers 1-3 and 2-3. The IR-active vibrational frequencies were calculated using DFT calculations at the BP86/TZVP level. Assignment of the CO-stretch mode descriptions is shown in the Figure below the table.

Table S2. The $\Delta \mathrm{G}$ values, which are the energy difference compared to the lowest energy configuration (1-2), dipole moments, $\mathrm{CO}$ - and $\mathrm{CN}$-stretch band frequencies and IR intensities of the isomers of 1 calculated using DFT BP86/TZVP.

Table S3. The $\Delta \mathrm{G}$ values, which are the energy difference of (fragment $+\mathrm{CN}$ ) compared to the lowest energy configuration (1-2), dipole moments, band frequencies and IR intensities of the CN-loss isomers of $\mathbf{1}$, calculated using DFT BP86/TZVP.

Table S4. The $\Delta \mathrm{G}$ values, which are the energy difference of (fragment $+\mathrm{CO}$ ) compared to the lowest energy configuration (1-2), dipole moments, band frequencies and IR intensities of the CO-loss isomers of $\mathbf{1}$, calculated using DFT BP86/TZVP.

Table S5. The $\Delta \mathrm{G}$ values, which are the energy difference of (fragment $+\mathrm{CN}$ ) compared to the lowest energy configuration (1-2), dipole moments, band frequencies and IR intensities of the $\mathrm{CN}$-loss isomers of $\mathbf{1}$, including a coordinated acetonitrile molecule, calculated using DFT BP86/TZVP.

Table S6. The $\Delta \mathrm{G}$ values, which are the energy difference of (fragment $+\mathrm{CO}$ ) compared to the lowest energy configuration (1-2), dipole moments, band frequencies and IR intensities of the CO-loss isomers of $\mathbf{1}$, including a coordinated acetonitrile molecule, calculated using DFT BP86/TZVP.

Table S7. The $\Delta \mathrm{G}$ values, which are the energy difference compared to the lowest energy configuration (2-1), dipole moments, and the band frequencies and IR intensities of the isomers of 2 calculated using DFT BP86/TZVP.

Table S8. The $\Delta \mathrm{G}$ values, which are the energy difference of (fragment $+\mathrm{CN}$ ) compared to the lowest energy configuration (2-1), dipole moments, and the band frequencies and IR intensities of the CN-loss isomers of 2, calculated using DFT BP86/TZVP. 
Table S9. The $\Delta \mathrm{G}$ values, which are the energy difference of (fragment $+\mathrm{CO}$ ) compared to the lowest energy configuration (2-1), dipole moments, and the band frequencies and IR intensities of the CO-loss isomers of $\mathbf{2}$, calculated using DFT BP86/TZVP.

Table S10. The $\Delta \mathrm{G}$ values, which are the energy difference of (fragment $+\mathrm{CN}$ ) compared to the lowest energy configuration (2-1), dipole moments, band frequencies and IR intensities of the $\mathrm{CN}$-loss isomers of $\mathbf{2}$, including a coordinated acetonitrile molecule, calculated using DFT BP86/TZVP.

Table S11. The $\Delta \mathrm{G}$ values, which are the energy difference of (fragment $+\mathrm{CO}$ ) compared to the lowest energy configuration (2-1), dipole moments, and the band frequencies and IR intensities of the CO-loss isomers of $\mathbf{2}$, including a coordinated acetonitrile molecule, calculated using DFT BP86/TZVP.

Table S12. Exponential decay fits of time-dependent data from seven wavelengths for $\mathbf{1}$. The average decay time $(\tau)$ is $28 \pm 11 \mathrm{ps}$.

Table S13. Exponential decay fits of time-dependent data from seven wavelengths for $\mathbf{2}$. The average decay time $(\tau)$ is $35 \pm 23 \mathrm{ps}$.

Figure S1. Exponential decay fits for seven wavelengths for 1 using $400 \mathrm{~nm}$ excitation.

Figure S2. (a) Time-resolved $400 \mathrm{~nm}$ excitation, IR probe spectra for $\mathbf{2}$ in $\mathrm{MeCN}(298 \mathrm{~K}$ ) at indicated time delays after excitation and (b) ground-state FTIR spectrum of $\mathbf{2}$ in room temperature $\mathrm{MeCN}$ solution.

Figure S3. Time-dependent scans for 2 in $\mathrm{MeCN}$ ( $298 \mathrm{~K}$ ) using $400 \mathrm{~nm}$ pump at (a) all selected wavenumbers, $(b-h)$ selected wavelengths. The red traces are a single-exponential decay with constant offset fits.

Figure S4. Long time-delay product spectrum extrapolated from the $286 \mathrm{ps}$ longest delay and the FTIR spectrum for $\mathbf{2}$ in $\mathrm{MeCN}$.

Figure S5. Calculated DFT spectra for all CN and CO-loss photoproduct isomers of 2: (a) CNloss isomers and $(\mathrm{b}, \mathrm{c}) \mathrm{CO}-$ loss isomers originating from all possible ground state isomeric structures. The feature at $1810 \mathrm{~cm}^{-1}$ in (b) corresponds to a bridge-bonded $\mathrm{Fe}-\mathrm{CO}-\mathrm{Fe}$ product. (d-f) are the same as (a-c) but with a coordinated acetonitrile molecule in the vacant position.

Figure S6. Long time UV-FTIR difference spectra for $\mathbf{2}$ at the indicated total UV-lamp exposure times.

Figure S7. Long time product spectrum extrapolated from $150 \mathrm{~s} \mathrm{UV} \mathrm{exposure} \mathrm{and} \mathrm{FTIR}$ spectrum for 2.

Figure S8. Long time UV-Vis and difference spectra for 2 dilute in $\mathrm{MeCN}$ with $60 \mathrm{~s}$ to $180 \mathrm{~s}$ Hg UV lamp exposure. 
Table S1. Symmetry assignments for ground state isomers 1-3 and 2-3. The IR-active vibrational frequencies were calculated using DFT calculations at the BP86/TZVP level. Assignment of the COstretch mode descriptions is shown in the Figure below the table.

\begin{tabular}{|c|c|c|l|}
\hline 1-3 $\mathbf{v}_{\mathrm{DFT}}\left(\mathbf{c m}^{\mathbf{- 1}}\right)$ & 2-3 $\mathbf{V}_{\mathrm{DFT}}\left(\mathbf{c m}^{\mathbf{- 1}}\right)$ & Symmetry & Description \\
\hline 1916 & 1916 & $\mathrm{~A}_{1}$ & $\begin{array}{l}\text { CO } 1 \text { and } 4 \text { are dominant stretches. CO 1 is } \\
\text { symmetric with 5. CO 2, 3, and 4 are antisymmetric } \\
\text { to CO 1 \& 5. }\end{array}$ \\
\hline 1924 & 1924 & $\mathrm{~A}_{1}$ & $\begin{array}{l}\text { CO } 1 \text { and } 4 \text { are dominant stretches. CO 2, 3, and 5 } \\
\text { are antisymmetric to 1 and 4. }\end{array}$ \\
\hline 1947 & 1947 & $\mathrm{~A}_{1}$ & $\begin{array}{l}\text { CO 2, 3, and 5 are dominant stretches. CO 1 and 3 } \\
\text { are symmetric. CO 2, 4, and 5 are also symmetric. } \\
\text { CO 1 and 3 are antisymmetric to CO 2, 4, and 5. }\end{array}$ \\
\hline 1972 & 1972 & $\mathrm{~A}_{1}$ & $\begin{array}{l}\text { CO 2, 4, and 5 are dominant stretches. CO 1 and 2 } \\
\text { are symmetric. CO 3, 4, and 5 are symmetric. CO 1 } \\
\text { and 2 are antisymmetric to CO 3, 4, and 5. }\end{array}$ \\
\hline 2010 & 2010 & $\mathrm{~A}_{1}$ & $\begin{array}{l}\text { CO 1 and 3 are dominant stretches. All CO stretches } \\
\text { are symmetric. }\end{array}$ \\
\hline 2116 & 2116 & $\mathrm{~A}_{1}$ & CN stretch \\
\hline
\end{tabular}
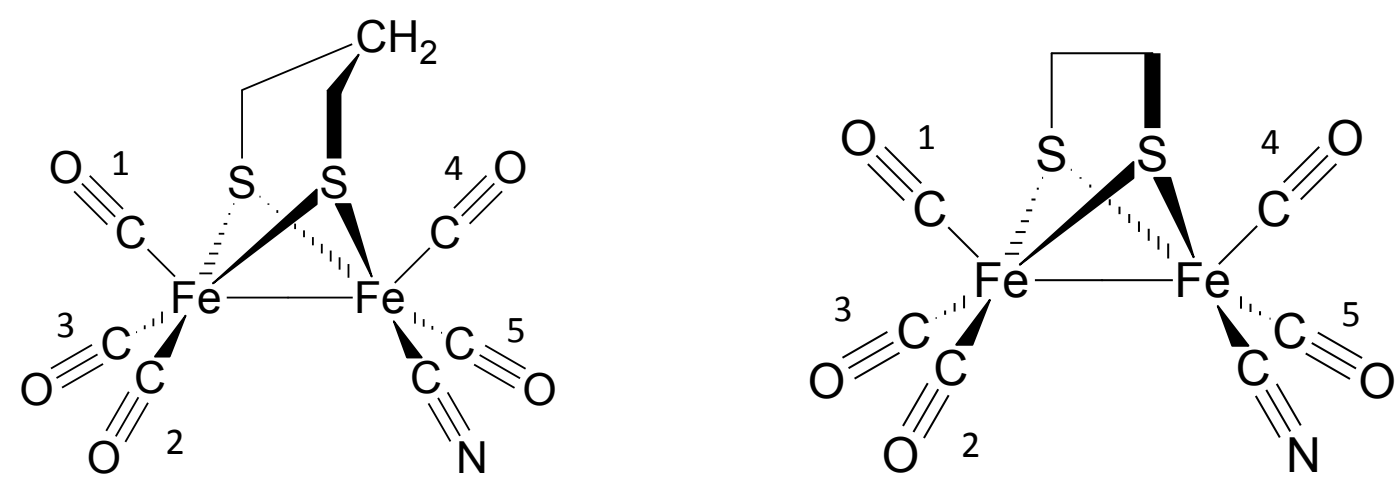
Table S2. The $\Delta \mathrm{G}$ values, which are the energy difference compared to the lowest energy configuration (1-2), dipole moments, $\mathrm{CO}$ - and $\mathrm{CN}$-stretch band frequencies and IR intensities of the isomers of 1 calculated using DFT BP86/TZVP.

\begin{tabular}{|c|c|c|c|c|}
\hline Isomer & $\Delta \mathrm{G}(\mathrm{kJ} / \mathrm{mol})$ & $\begin{array}{c}\text { Dipole moment } \\
\text { (Debye) }\end{array}$ & $\begin{array}{l}\text { Band Frequency } \\
\left(\mathrm{cm}^{-1}\right)\end{array}$ & $\begin{array}{l}\text { Band Intensity } \\
\text { (arb. Units) }\end{array}$ \\
\hline \multirow[t]{6}{*}{$1-1$} & \multirow[t]{6}{*}{5.2} & \multirow[t]{6}{*}{6.23} & 1923 & 684 \\
\hline & & & 1927 & 55 \\
\hline & & & 1946 & 1255 \\
\hline & & & 1965 & 1139 \\
\hline & & & 2008 & 961 \\
\hline & & & 2106 & 62 \\
\hline \multirow[t]{6}{*}{$1-2$} & \multirow[t]{6}{*}{0.00} & \multirow[t]{6}{*}{5.94} & 1923 & 655 \\
\hline & & & 1924 & 214 \\
\hline & & & 1947 & 1136 \\
\hline & & & 1972 & 1207 \\
\hline & & & 2007 & 972 \\
\hline & & & 2100 & 57 \\
\hline \multirow[t]{6}{*}{$1-3$} & \multirow[t]{6}{*}{10.4} & \multirow[t]{6}{*}{9.25} & 1917 & 513 \\
\hline & & & 1924 & 852 \\
\hline & & & 1947 & 740 \\
\hline & & & 1972 & 1313 \\
\hline & & & 2010 & 682 \\
\hline & & & 2116 & 52 \\
\hline \multirow[t]{6}{*}{$1-4$} & \multirow[t]{6}{*}{12.7} & \multirow[t]{6}{*}{9.23} & 1916 & 543 \\
\hline & & & 1924 & 817 \\
\hline & & & 1948 & 704 \\
\hline & & & 1972 & 1342 \\
\hline & & & 2010 & 685 \\
\hline & & & 2115 & 50 \\
\hline
\end{tabular}


Table S3. The $\Delta \mathrm{G}$ values, which are the energy difference of (fragment $+\mathrm{CN}$ ) compared to the lowest energy configuration (1-2), dipole moments, band frequencies and IR intensities of the CN-loss isomers of 1 , calculated using DFT BP86/TZVP.

\begin{tabular}{|c|c|c|c|c|}
\hline Isomer & $\Delta \mathrm{G}(\mathrm{kJ} / \mathrm{mol})$ & $\begin{array}{l}\text { Dipole Moment } \\
\text { (Debye) }\end{array}$ & $\begin{array}{l}\text { band frequency } \\
\qquad\left(\mathrm{cm}^{-1}\right)\end{array}$ & $\begin{array}{l}\text { band intensity } \\
\text { (arb. Units) }\end{array}$ \\
\hline \multirow[t]{5}{*}{$5 C O 1-1$} & \multirow[t]{5}{*}{298.6} & \multirow[t]{5}{*}{5.59} & 1953 & 431 \\
\hline & & & 1979 & 201 \\
\hline & & & 1995 & 1600 \\
\hline & & & 1997 & 757 \\
\hline & & & 2055 & 638 \\
\hline \multirow[t]{5}{*}{$5 C O 1-2$} & \multirow[t]{5}{*}{301.0} & \multirow[t]{5}{*}{5.74} & 1951 & 449 \\
\hline & & & 1979 & 164 \\
\hline & & & 1994 & 1638 \\
\hline & & & 1998 & 744 \\
\hline & & & 2055 & 656 \\
\hline \multirow[t]{5}{*}{$5 C O 1-3$} & \multirow[t]{5}{*}{281.6} & \multirow[t]{5}{*}{4.61} & 1950 & 562 \\
\hline & & & 1976 & 616 \\
\hline & & & 1983 & 610 \\
\hline & & & 1994 & 1571 \\
\hline & & & 2040 & 657 \\
\hline \multirow[t]{5}{*}{$5 C O 1-4$} & \multirow[t]{5}{*}{275.2} & \multirow[t]{5}{*}{4.62} & 1948 & 584 \\
\hline & & & 1977 & 593 \\
\hline & & & 1984 & 594 \\
\hline & & & 1992 & 1557 \\
\hline & & & 2041 & 675 \\
\hline
\end{tabular}


Table S4. The $\Delta G$ values, which are the energy difference of (fragment $+\mathrm{CO}$ ) compared to the lowest energy configuration (1-2), dipole moments, band frequencies and IR intensities of the CO-loss isomers of 1 , calculated using DFT BP86/TZVP.

\begin{tabular}{|c|c|c|c|c|}
\hline Isomer & $\Delta \mathrm{G}(\mathrm{kJ} / \mathrm{mol})$ & $\begin{array}{l}\text { Dipole Moment } \\
\text { (Debye) }\end{array}$ & $\begin{array}{l}\text { band frequency } \\
\left(\mathrm{cm}^{-1}\right)\end{array}$ & $\begin{array}{l}\text { band intensity } \\
\text { (arb. Units) }\end{array}$ \\
\hline \multirow[t]{5}{*}{$1-1.1$} & \multirow[t]{5}{*}{160.1} & \multirow[t]{5}{*}{7.05} & 1895 & 494 \\
\hline & & & 1934 & 920 \\
\hline & & & 1939 & 1120 \\
\hline & & & 1990 & 997 \\
\hline & & & 2102 & 50 \\
\hline \multirow[t]{5}{*}{$1-1.2$} & \multirow[t]{5}{*}{144.5} & \multirow[t]{5}{*}{6.03} & 1886 & 799 \\
\hline & & & 1923 & 675 \\
\hline & & & 1930 & 1324 \\
\hline & & & 1977 & 1010 \\
\hline & & & 2104 & 59 \\
\hline \multirow[t]{5}{*}{$1-1.3$} & \multirow[t]{5}{*}{152.9} & \multirow[t]{5}{*}{6.24} & 1910 & 69 \\
\hline & & & 1915 & 1753 \\
\hline & & & 1926 & 796 \\
\hline & & & 1985 & 1083 \\
\hline & & & 2063 & 61 \\
\hline \multirow[t]{5}{*}{$1-1.4$} & \multirow[t]{5}{*}{144.3} & \multirow[t]{5}{*}{6.03} & 1886 & 798 \\
\hline & & & 1923 & 673 \\
\hline & & & 1930 & 1327 \\
\hline & & & 1977 & 1010 \\
\hline & & & 2104 & 60 \\
\hline \multirow[t]{5}{*}{$1-1.5$} & \multirow[t]{5}{*}{153.0} & \multirow[t]{5}{*}{6.25} & 1910 & 99 \\
\hline & & & 1915 & 1717 \\
\hline & & & 1926 & 802 \\
\hline & & & 1985 & 1083 \\
\hline & & & 2063 & 61 \\
\hline \multirow[t]{5}{*}{$1-2.1$} & \multirow[t]{5}{*}{152.6} & \multirow[t]{5}{*}{6.37} & 1897 & 449 \\
\hline & & & 1931 & 962 \\
\hline & & & 1939 & 1150 \\
\hline & & & 1988 & 1017 \\
\hline & & & 2096 & 45 \\
\hline \multirow[t]{5}{*}{$1-2.2$} & \multirow[t]{5}{*}{148.7} & \multirow[t]{5}{*}{5.73} & 1888 & 794 \\
\hline & & & 1919 & 684 \\
\hline & & & 1929 & 1376 \\
\hline & & & 1975 & 1056 \\
\hline & & & 2097 & 56 \\
\hline \multirow[t]{5}{*}{$1-2.3$} & \multirow[t]{5}{*}{139.4} & \multirow[t]{5}{*}{5.15} & 1813 & 497 \\
\hline & & & 1909 & 750 \\
\hline & & & 1938 & 1441 \\
\hline & & & 1968 & 936 \\
\hline & & & 2085 & 39 \\
\hline
\end{tabular}




\begin{tabular}{|c|c|c|c|c|}
\hline \multirow[t]{5}{*}{$1-2.4$} & \multirow[t]{5}{*}{148.8} & \multirow[t]{5}{*}{5.74} & 1887 & 796 \\
\hline & & & 1919 & 684 \\
\hline & & & 1929 & 1371 \\
\hline & & & 1974 & 1058 \\
\hline & & & 2097 & 56 \\
\hline \multirow[t]{5}{*}{$1-2.5$} & \multirow[t]{5}{*}{139.0} & \multirow[t]{5}{*}{5.16} & 1813 & 498 \\
\hline & & & 1909 & 751 \\
\hline & & & 1938 & 1445 \\
\hline & & & 1967 & 930 \\
\hline & & & 2085 & 39 \\
\hline \multirow[t]{5}{*}{$1-3.1$} & \multirow[t]{5}{*}{167.4} & \multirow[t]{5}{*}{10.23} & 1901 & 562 \\
\hline & & & 1920 & 708 \\
\hline & & & 1949 & 1644 \\
\hline & & & 1985 & 623 \\
\hline & & & 2109 & 31 \\
\hline \multirow[t]{5}{*}{$1-3.2$} & \multirow[t]{5}{*}{145.7} & \multirow[t]{5}{*}{8.91} & 1887 & 820 \\
\hline & & & 1913 & 758 \\
\hline & & & 1935 & 1569 \\
\hline & & & 1974 & 713 \\
\hline & & & 2107 & 56 \\
\hline \multirow[t]{5}{*}{$1-3.3$} & \multirow[t]{5}{*}{152.5} & \multirow[t]{5}{*}{9.25} & 1889 & 1038 \\
\hline & & & 1913 & 798 \\
\hline & & & 1943 & 1562 \\
\hline & & & 1969 & 466 \\
\hline & & & 2111 & 66 \\
\hline \multirow[t]{5}{*}{$1-3.4$} & \multirow[t]{5}{*}{183.4} & \multirow[t]{5}{*}{9.30} & 1908 & 543 \\
\hline & & & 1924 & 1149 \\
\hline & & & 1952 & 751 \\
\hline & & & 2007 & 853 \\
\hline & & & 2068 & 56 \\
\hline \multirow[t]{5}{*}{$1-3.5$} & \multirow[t]{5}{*}{158.8} & \multirow[t]{5}{*}{9.21} & 1896 & 1253 \\
\hline & & & 1912 & 804 \\
\hline & & & 1935 & 695 \\
\hline & & & 1991 & 877 \\
\hline & & & 2076 & 43 \\
\hline \multirow[t]{5}{*}{$1-4.1$} & \multirow[t]{5}{*}{141.4} & \multirow[t]{5}{*}{8.93} & 1803 & 383 \\
\hline & & & 1917 & 670 \\
\hline & & & 1940 & 1645 \\
\hline & & & 1975 & 892 \\
\hline & & & 2084 & 31 \\
\hline $1-4.2$ & 154.6 & 9.32 & 1896 & 1240 \\
\hline & & & 1911 & 787 \\
\hline & & & 1931 & 686 \\
\hline & & & 1988 & 878 \\
\hline & & & 2077 & 44 \\
\hline
\end{tabular}




\begin{tabular}{|c|c|c|c|c|}
\hline \multirow[t]{5}{*}{$1-4.3$} & \multirow[t]{5}{*}{161.9} & \multirow[t]{5}{*}{9.43} & 1889 & 1115 \\
\hline & & & 1911 & 785 \\
\hline & & & 1944 & 1506 \\
\hline & & & 1970 & 495 \\
\hline & & & 2110 & 64 \\
\hline \multirow[t]{5}{*}{$1-4.4$} & \multirow[t]{5}{*}{165.9} & \multirow[t]{5}{*}{10.20} & 1903 & 518 \\
\hline & & & 1919 & 738 \\
\hline & & & 1950 & 1643 \\
\hline & & & 1984 & 599 \\
\hline & & & 2108 & 28 \\
\hline \multirow[t]{5}{*}{$1-4.5$} & \multirow[t]{5}{*}{154.8} & \multirow[t]{5}{*}{9.11} & 1888 & 859 \\
\hline & & & 1912 & 728 \\
\hline & & & 1935 & 1585 \\
\hline & & & 1974 & 734 \\
\hline & & & 2108 & 57 \\
\hline
\end{tabular}


Table S5. The $\Delta \mathrm{G}$ values, which are the energy difference of (fragment $+\mathrm{CN}$ ) compared to the lowest energy configuration (1-2), dipole moments, band frequencies and IR intensities of the CN-loss isomers of $\mathbf{1}$, including a coordinated acetonitrile molecule, calculated using DFT BP86/TZVP.

\begin{tabular}{|c|c|c|c|c|}
\hline Isomer & $\Delta \mathrm{G}(\mathrm{kJ} / \mathrm{mol})$ & $\begin{array}{l}\text { Dipole Moment } \\
\text { (Debye) }\end{array}$ & $\begin{array}{l}\text { band frequency } \\
\qquad\left(\mathrm{cm}^{-1}\right)\end{array}$ & $\begin{array}{l}\text { band intensity } \\
\text { (arb. Units) }\end{array}$ \\
\hline \multirow[t]{5}{*}{$5 C O 1-1$} & \multirow[t]{5}{*}{230.0} & \multirow[t]{5}{*}{9.78} & 1957 & 212 \\
\hline & & & 1967 & 379 \\
\hline & & & 1986 & 943 \\
\hline & & & 1993 & 1063 \\
\hline & & & 2043 & 990 \\
\hline \multirow[t]{5}{*}{$5 C O 1-2$} & \multirow[t]{5}{*}{221.1} & \multirow[t]{5}{*}{9.81} & 1956 & 251 \\
\hline & & & 1968 & 369 \\
\hline & & & 1987 & 903 \\
\hline & & & 1992 & 1118 \\
\hline & & & 2044 & 1002 \\
\hline \multirow[t]{5}{*}{$5 C O 1-3$} & \multirow[t]{5}{*}{214.0} & \multirow[t]{5}{*}{5.18} & 1952 & 429 \\
\hline & & & 1960 & 697 \\
\hline & & & 1970 & 580 \\
\hline & & & 1994 & 1408 \\
\hline & & & 2030 & 613 \\
\hline \multirow[t]{5}{*}{$5 C O 1-4$} & \multirow[t]{5}{*}{212.4} & \multirow[t]{5}{*}{5.08} & 1951 & 459 \\
\hline & & & 1961 & 693 \\
\hline & & & 1971 & 541 \\
\hline & & & 1993 & 1394 \\
\hline & & & 2030 & 639 \\
\hline
\end{tabular}


Table S6. The $\Delta G$ values, which are the energy difference of (fragment $+\mathrm{CO}$ ) compared to the lowest energy configuration (1-2), dipole moments, band frequencies and IR intensities of the CO-loss isomers of $\mathbf{1}$, including a coordinated acetonitrile molecule, calculated using DFT BP86/TZVP.

\begin{tabular}{|c|c|c|c|c|}
\hline Isomer & $\Delta \mathrm{G}(\mathrm{kJ} / \mathrm{mol})$ & $\begin{array}{l}\text { Dipole Moment } \\
\text { (Debye) }\end{array}$ & $\begin{array}{l}\text { band frequency } \\
\left(\mathrm{cm}^{-1}\right)\end{array}$ & $\begin{array}{l}\text { band intensity } \\
\text { (arb. Units) }\end{array}$ \\
\hline \multirow[t]{5}{*}{$1-1.1$} & \multirow[t]{5}{*}{119.2} & \multirow[t]{5}{*}{13.51} & 1905 & 142 \\
\hline & & & 1928 & 1202 \\
\hline & & & 1939 & 538 \\
\hline & & & 1982 & 1458 \\
\hline & & & 2102 & 84 \\
\hline \multirow[t]{5}{*}{$1-1.2$} & \multirow[t]{5}{*}{116.1} & \multirow[t]{5}{*}{9.10} & 1897 & 664 \\
\hline & & & 1902 & 623 \\
\hline & & & 1934 & 1145 \\
\hline & & & 1966 & 901 \\
\hline & & & 2101 & 80 \\
\hline \multirow[t]{5}{*}{$1-1.3$} & \multirow[t]{5}{*}{115.8} & \multirow[t]{5}{*}{5.19} & 1905 & 780 \\
\hline & & & 1916 & 334 \\
\hline & & & 1926 & 1210 \\
\hline & & & 1978 & 1071 \\
\hline & & & 2090 & 87 \\
\hline \multirow[t]{5}{*}{$1-1.4$} & \multirow[t]{5}{*}{116.1} & \multirow[t]{5}{*}{9.10} & 1897 & 664 \\
\hline & & & 1902 & 624 \\
\hline & & & 1934 & 1146 \\
\hline & & & 1966 & 901 \\
\hline & & & 2101 & 80 \\
\hline \multirow[t]{5}{*}{$1-1.5$} & \multirow[t]{5}{*}{116.3} & \multirow[t]{5}{*}{5.19} & 1905 & 780 \\
\hline & & & 1916 & 326 \\
\hline & & & 1926 & 1219 \\
\hline & & & 1978 & 1070 \\
\hline & & & 2090 & 87 \\
\hline \multirow[t]{5}{*}{$1-2.1$} & \multirow[t]{5}{*}{127.1} & \multirow[t]{5}{*}{13.40} & 1906 & 21 \\
\hline & & & 1924 & 1327 \\
\hline & & & 1939 & 507 \\
\hline & & & 1979 & 1496 \\
\hline & & & 2095 & 77 \\
\hline \multirow[t]{5}{*}{$1-2.2$} & \multirow[t]{5}{*}{115.3} & \multirow[t]{5}{*}{9.11} & 1896 & 637 \\
\hline & & & 1900 & 675 \\
\hline & & & 1933 & 1185 \\
\hline & & & 1965 & 927 \\
\hline & & & 2095 & 74 \\
\hline \multirow[t]{5}{*}{$1-2.3$} & \multirow[t]{5}{*}{117.6} & \multirow[t]{5}{*}{4.51} & 1826 & 479 \\
\hline & & & 1903 & 720 \\
\hline & & & 1943 & 1345 \\
\hline & & & 1964 & 893 \\
\hline & & & 2098 & 65 \\
\hline
\end{tabular}




\begin{tabular}{|c|c|c|c|c|}
\hline \multirow[t]{5}{*}{$1-2.4$} & \multirow[t]{5}{*}{115.3} & \multirow[t]{5}{*}{9.11} & 1896 & 635 \\
\hline & & & 1900 & 676 \\
\hline & & & 1933 & 1186 \\
\hline & & & 1965 & 927 \\
\hline & & & 2095 & 74 \\
\hline \multirow[t]{5}{*}{$1-2.5$} & \multirow[t]{5}{*}{108.6} & \multirow[t]{5}{*}{4.52} & 1826 & 478 \\
\hline & & & 1903 & 721 \\
\hline & & & 1943 & 1348 \\
\hline & & & 1964 & 892 \\
\hline & & & 2098 & 65 \\
\hline \multirow[t]{5}{*}{ 1-3.1 } & \multirow[t]{5}{*}{127.4} & \multirow[t]{5}{*}{15.07} & 1907 & 338 \\
\hline & & & 1918 & 914 \\
\hline & & & 1953 & 1100 \\
\hline & & & 1979 & 1002 \\
\hline & & & 2111 & 74 \\
\hline \multirow[t]{5}{*}{$1-3.2$} & \multirow[t]{5}{*}{105.0} & \multirow[t]{5}{*}{5.30} & 1893 & 583 \\
\hline & & & 1905 & 823 \\
\hline & & & 1935 & 1426 \\
\hline & & & 1966 & 785 \\
\hline & & & 2089 & 97 \\
\hline \multirow[t]{5}{*}{$1-3.3$} & \multirow[t]{5}{*}{120.3} & \multirow[t]{5}{*}{9.86} & 1895 & 713 \\
\hline & & & 1900 & 933 \\
\hline & & & 1940 & 1302 \\
\hline & & & 1965 & 456 \\
\hline & & & 2109 & 73 \\
\hline \multirow[t]{5}{*}{$1-3.4$} & \multirow[t]{5}{*}{140.5} & \multirow[t]{5}{*}{10.82} & 1911 & 442 \\
\hline & & & 1923 & 882 \\
\hline & & & 1946 & 953 \\
\hline & & & 1999 & 1148 \\
\hline & & & 2100 & 80 \\
\hline \multirow[t]{5}{*}{$1-3.5$} & \multirow[t]{5}{*}{120.3} & \multirow[t]{5}{*}{7.78} & 1901 & 976 \\
\hline & & & 1907 & 848 \\
\hline & & & 1922 & 637 \\
\hline & & & 1983 & 930 \\
\hline & & & 2103 & 57 \\
\hline \multirow[t]{5}{*}{$1-4.1$} & \multirow[t]{5}{*}{134.4} & \multirow[t]{5}{*}{11.20} & 1910 & 538 \\
\hline & & & 1920 & 732 \\
\hline & & & 1948 & 1054 \\
\hline & & & 1999 & 1137 \\
\hline & & & 2101 & 81 \\
\hline $1-4.2$ & 121.3 & 7.83 & 1900 & 1006 \\
\hline & & & 1906 & 775 \\
\hline & & & 1920 & 614 \\
\hline & & & 1983 & 975 \\
\hline & & & 2104 & 53 \\
\hline
\end{tabular}




\begin{tabular}{|c|c|c|c|c|}
\hline \multirow[t]{5}{*}{$1-4.3$} & \multirow{5}{*}{108.7} & \multirow[t]{5}{*}{5.59} & 1894 & 610 \\
\hline & & & 1904 & 813 \\
\hline & & & 1936 & 1409 \\
\hline & & & 1967 & 794 \\
\hline & & & 2089 & 95 \\
\hline \multirow[t]{5}{*}{$1-4.4$} & \multirow[t]{5}{*}{132.9} & \multirow[t]{5}{*}{15.16} & 1905 & 399 \\
\hline & & & 1920 & 816 \\
\hline & & & 1954 & 1099 \\
\hline & & & 1978 & 939 \\
\hline & & & 2111 & 73 \\
\hline \multirow[t]{5}{*}{$1-4.5$} & \multirow[t]{5}{*}{124.7} & \multirow[t]{5}{*}{9.98} & 1894 & 731 \\
\hline & & & 1899 & 974 \\
\hline & & & 1939 & 1196 \\
\hline & & & 1967 & 513 \\
\hline & & & 2108 & 71 \\
\hline
\end{tabular}


Table S7. The $\Delta \mathrm{G}$ values, which are the energy difference compared to the lowest energy configuration (2-1), dipole moments, and the band frequencies and IR intensities of the isomers of $\mathbf{2}$ calculated using DFT BP86/TZVP.

\begin{tabular}{|c|c|c|c|c|}
\hline Isomer & $\Delta \mathrm{G}(\mathrm{kJ} / \mathrm{mol})$ & $\begin{array}{l}\text { Dipole moment } \\
\text { (Debye) }\end{array}$ & $\begin{array}{l}\text { Band Frequency } \\
\qquad\left(\mathrm{cm}^{-1}\right)\end{array}$ & $\begin{array}{l}\text { Band Intensity } \\
\text { (arb. Units) }\end{array}$ \\
\hline \multirow[t]{6}{*}{$2-1$} & \multirow[t]{6}{*}{0} & \multirow[t]{6}{*}{5.79} & 1925 & 128 \\
\hline & & & 1927 & 667 \\
\hline & & & 1947 & 1236 \\
\hline & & & 1962 & 1258 \\
\hline & & & 2008 & 904 \\
\hline & & & 2107 & 60 \\
\hline \multirow[t]{6}{*}{$2-3$} & \multirow[t]{6}{*}{9.0} & \multirow[t]{6}{*}{8.43} & 1919 & 456 \\
\hline & & & 1928 & 904 \\
\hline & & & 1949 & 812 \\
\hline & & & 1973 & 1332 \\
\hline & & & 2012 & 647 \\
\hline & & & 2117 & 55 \\
\hline
\end{tabular}


Table S8. The $\Delta \mathrm{G}$ values, which are the energy difference of (fragment $+\mathrm{CN}$ ) compared to the lowest energy configuration (2-1), dipole moments, and the band frequencies and IR intensities of the CN-loss isomers of 2, calculated using DFT BP86/TZVP.

\begin{tabular}{|c|c|c|c|c|}
\hline Isomer & $\Delta \mathrm{G}(\mathrm{kJ} / \mathrm{mol})$ & $\begin{array}{l}\text { Dipole moment } \\
\text { (Debye) }\end{array}$ & $\begin{array}{l}\text { Band Frequency } \\
\qquad\left(\mathrm{cm}^{-1}\right)\end{array}$ & $\begin{array}{l}\text { Band Intensity } \\
\text { (arb. Units) }\end{array}$ \\
\hline \multirow[t]{5}{*}{$5 C O 2-1$} & \multirow[t]{5}{*}{305.6} & \multirow[t]{5}{*}{5.04} & 1954 & 426 \\
\hline & & & 1982 & 144 \\
\hline & & & 1997 & 1678 \\
\hline & & & 1999 & 798 \\
\hline & & & 2057 & 597 \\
\hline \multirow[t]{5}{*}{$5 C O 2-3$} & \multirow[t]{5}{*}{292.8} & \multirow[t]{5}{*}{3.71} & 1954 & 559 \\
\hline & & & 1981 & 601 \\
\hline & & & 1985 & 623 \\
\hline & & & 1997 & 1672 \\
\hline & & & 2043 & 584 \\
\hline
\end{tabular}


Table S9. The $\Delta G$ values, which are the energy difference of (fragment $+\mathrm{CO}$ ) compared to the lowest energy configuration (2-1), dipole moments, and the band frequencies and IR intensities of the CO-loss isomers of 2, calculated using DFT BP86/TZVP.

\begin{tabular}{|c|c|c|c|c|}
\hline Isomer & $\Delta \mathrm{G}(\mathrm{kJ} / \mathrm{mol})$ & $\begin{array}{l}\text { Dipole Moment } \\
\text { (Debye) }\end{array}$ & $\begin{array}{l}\text { band frequency } \\
\left(\mathrm{cm}^{-1}\right)\end{array}$ & $\begin{array}{l}\text { band intensity } \\
\text { (arb. Units) }\end{array}$ \\
\hline \multirow[t]{5}{*}{$2-1.1$} & \multirow[t]{5}{*}{156.6} & \multirow[t]{5}{*}{6.22} & 1898 & 442 \\
\hline & & & 1932 & 1022 \\
\hline & & & 1939 & 1100 \\
\hline & & & 1989 & 978 \\
\hline & & & 2100 & 43 \\
\hline \multirow[t]{5}{*}{$2-1.2$} & \multirow[t]{5}{*}{161.1} & \multirow[t]{5}{*}{5.63} & 1891 & 809 \\
\hline & & & 1922 & 733 \\
\hline & & & 1931 & 1395 \\
\hline & & & 1977 & 954 \\
\hline & & & 2106 & 60 \\
\hline \multirow[t]{5}{*}{$2-1.3$} & \multirow[t]{5}{*}{157.3} & \multirow[t]{5}{*}{4.93} & 1812 & 530 \\
\hline & & & 1909 & 802 \\
\hline & & & 1937 & 1261 \\
\hline & & & 1970 & 993 \\
\hline & & & 2089 & 31 \\
\hline \multirow[t]{5}{*}{$2-1.4$} & \multirow[t]{5}{*}{161.0} & \multirow[t]{5}{*}{5.62} & 1890 & 809 \\
\hline & & & 1922 & 732 \\
\hline & & & 1931 & 1402 \\
\hline & & & 1976 & 953 \\
\hline & & & 2106 & 60 \\
\hline \multirow[t]{5}{*}{$2-1.5$} & \multirow[t]{5}{*}{157.0} & \multirow[t]{5}{*}{4.92} & 1812 & 530 \\
\hline & & & 1909 & 799 \\
\hline & & & 1937 & 1262 \\
\hline & & & 1970 & 995 \\
\hline & & & 2089 & 31 \\
\hline \multirow[t]{5}{*}{$2-3.1$} & \multirow[t]{5}{*}{168.5} & \multirow[t]{5}{*}{9.34} & 1903 & 525 \\
\hline & & & 1921 & 751 \\
\hline & & & 1950 & 1619 \\
\hline & & & 1985 & 597 \\
\hline & & & 2109 & 31 \\
\hline \multirow[t]{5}{*}{$2-3.2$} & \multirow[t]{5}{*}{134.3} & \multirow[t]{5}{*}{5.83} & 1900 & 663 \\
\hline & & & 1907 & 927 \\
\hline & & & 1935 & 1028 \\
\hline & & & 1951 & 875 \\
\hline & & & 1976 & 463 \\
\hline \multirow[t]{5}{*}{$2-3.3$} & \multirow[t]{5}{*}{169.6} & \multirow[t]{5}{*}{8.36} & 1893 & 1135 \\
\hline & & & 1915 & 789 \\
\hline & & & 1944 & 1621 \\
\hline & & & 1972 & 429 \\
\hline & & & 2111 & 61 \\
\hline
\end{tabular}




\begin{tabular}{|c|c|c|c|c|}
\hline \multirow[t]{5}{*}{$2-3.4$} & \multirow[t]{5}{*}{186.6} & \multirow[t]{5}{*}{8.57} & 1907 & 599 \\
\hline & & & 1926 & 1152 \\
\hline & & & 1954 & 736 \\
\hline & & & 2008 & 819 \\
\hline & & & 2069 & 53 \\
\hline \multirow[t]{5}{*}{$2-3.5$} & \multirow[t]{5}{*}{163.5} & \multirow[t]{5}{*}{8.34} & 1900 & 1296 \\
\hline & & & 1916 & 849 \\
\hline & & & 1936 & 712 \\
\hline & & & 1992 & 842 \\
\hline & & & 2077 & 39 \\
\hline
\end{tabular}


Table S10. The $\Delta G$ values, which are the energy difference of (fragment $+\mathrm{CN}$ ) compared to the lowest energy configuration (2-1), dipole moments, band frequencies and IR intensities of the CN-loss isomers of 2, including a coordinated acetonitrile molecule, calculated using DFT BP86/TZVP.

\begin{tabular}{|c|c|c|c|c|}
\hline Isomer & $\Delta \mathrm{G}(\mathrm{kJ} / \mathrm{mol})$ & $\begin{array}{l}\text { Dipole moment } \\
\text { (Debye) }\end{array}$ & $\begin{array}{l}\text { Band Frequency } \\
\qquad\left(\mathrm{cm}^{-1}\right)\end{array}$ & $\begin{array}{l}\text { Band Intensity } \\
\text { (arb. Units) }\end{array}$ \\
\hline \multirow[t]{5}{*}{$5 C O 2-1$} & \multirow[t]{5}{*}{218.6} & \multirow[t]{5}{*}{9.51} & 1958 & 228 \\
\hline & & & 1971 & 310 \\
\hline & & & 1988 & 967 \\
\hline & & & 1994 & 1187 \\
\hline & & & 2045 & 954 \\
\hline \multirow[t]{5}{*}{$5 C O 2-3$} & \multirow[t]{5}{*}{214.2} & \multirow[t]{5}{*}{5.16} & 1955 & 407 \\
\hline & & & 1963 & 681 \\
\hline & & & 1973 & 625 \\
\hline & & & 1996 & 1468 \\
\hline & & & 2032 & 587 \\
\hline
\end{tabular}


Table S11. The $\Delta G$ values, which are the energy difference of (fragment $+\mathrm{CO}$ ) compared to the lowest energy configuration (2-1), dipole moments, and the band frequencies and IR intensities of the CO-loss isomers of $\mathbf{2}$, including a coordinated acetonitrile molecule, calculated using DFT BP86/TZVP.

\begin{tabular}{|c|c|c|c|c|}
\hline Isomer & $\Delta \mathrm{G}(\mathrm{kJ} / \mathrm{mol})$ & $\begin{array}{c}\text { Dipole Moment } \\
\text { (Debye) }\end{array}$ & $\begin{array}{l}\text { band frequency } \\
\left(\mathrm{cm}^{-1}\right)\end{array}$ & $\begin{array}{l}\text { band intensity } \\
\text { (arb. Units) }\end{array}$ \\
\hline \multirow[t]{5}{*}{$2-1.1$} & \multirow[t]{5}{*}{118.6} & \multirow[t]{5}{*}{13.25} & 1906 & 61 \\
\hline & & & 1926 & 1339 \\
\hline & & & 1938 & 521 \\
\hline & & & 1980 & 1458 \\
\hline & & & 2102 & 80 \\
\hline \multirow[t]{5}{*}{$2-1.2$} & \multirow[t]{5}{*}{116.0} & \multirow[t]{5}{*}{9.35} & 1898 & 613 \\
\hline & & & 1901 & 693 \\
\hline & & & 1934 & 1228 \\
\hline & & & 1965 & 890 \\
\hline & & & 2102 & 78 \\
\hline \multirow[t]{5}{*}{$2-1.3$} & \multirow[t]{5}{*}{114.7} & \multirow[t]{5}{*}{5.48} & 1908 & 755 \\
\hline & & & 1917 & 212 \\
\hline & & & 1924 & 1421 \\
\hline & & & 1979 & 1072 \\
\hline & & & 2091 & 90 \\
\hline \multirow[t]{5}{*}{$2-1.4$} & \multirow[t]{5}{*}{116.0} & \multirow[t]{5}{*}{9.35} & 1898 & 612 \\
\hline & & & 1901 & 694 \\
\hline & & & 1934 & 1229 \\
\hline & & & 1965 & 890 \\
\hline & & & 2102 & 78 \\
\hline \multirow[t]{5}{*}{$2-1.5$} & \multirow[t]{5}{*}{114.9} & \multirow[t]{5}{*}{5.48} & 1908 & 756 \\
\hline & & & 1917 & 204 \\
\hline & & & 1924 & 1429 \\
\hline & & & 1979 & 1070 \\
\hline & & & 2091 & 90 \\
\hline \multirow[t]{5}{*}{$2-3.1$} & \multirow[t]{5}{*}{127.0} & \multirow[t]{5}{*}{14.72} & 1908 & 300 \\
\hline & & & 1920 & 975 \\
\hline & & & 1954 & 1130 \\
\hline & & & 1979 & 951 \\
\hline & & & 2112 & 78 \\
\hline \multirow[t]{5}{*}{$2-3.2$} & \multirow[t]{5}{*}{105.0} & \multirow[t]{5}{*}{5.01} & 1896 & 525 \\
\hline & & & 1907 & 861 \\
\hline & & & 1936 & 1529 \\
\hline & & & 1968 & 767 \\
\hline & & & 2090 & 98 \\
\hline \multirow[t]{5}{*}{$2-3.3$} & \multirow[t]{5}{*}{122.0} & \multirow[t]{5}{*}{9.83} & 1896 & 702 \\
\hline & & & 1903 & 1000 \\
\hline & & & 1941 & 1290 \\
\hline & & & 1968 & 477 \\
\hline & & & 2110 & 76 \\
\hline
\end{tabular}




\begin{tabular}{|c|c|c|c|c|}
\hline \multirow[t]{5}{*}{$2-3.4$} & \multirow[t]{5}{*}{132.0} & \multirow[t]{5}{*}{10.37} & 1913 & 234 \\
\hline & & & 1922 & 1169 \\
\hline & & & 1948 & 958 \\
\hline & & & 2001 & 1120 \\
\hline & & & 2101 & 86 \\
\hline \multirow[t]{5}{*}{ 2-3.5 } & \multirow[t]{5}{*}{120.2} & \multirow[t]{5}{*}{7.59} & 1904 & 975 \\
\hline & & & 1909 & 887 \\
\hline & & & 1924 & 673 \\
\hline & & & 1986 & 920 \\
\hline & & & 2104 & 60 \\
\hline
\end{tabular}


Table S12. Exponential decay fits of time-dependent data from seven wavelengths for 1 . The average decay time $(\tau)$ is $28 \pm 11 \mathrm{ps}$.

\begin{tabular}{|c|c|c|c|}
\hline Wavelength $\left(\mathrm{cm}^{-1}\right)$ & Amplitude ( $\triangle O D)$ & $\tau(p s)$ & Offset ( $\triangle O D)$ \\
\hline 2038 & -0.0057 & 22 & 0.00101 \\
\hline 2022 & 0.0014 & 50 & 0.00053 \\
\hline 1998 & -0.0010 & 28 & 0.00014 \\
\hline 1990 & -0.0016 & 31 & -0.00028 \\
\hline 1979 & -0.0160 & 17 & -0.01167 \\
\hline 1943 & 0.0024 & 27 & -0.00035 \\
\hline 1925 & 0.0021 & 19 & -0.00110 \\
\hline
\end{tabular}


Table S13. Exponential decay fits of time-dependent data from seven wavelengths for 2 . The average decay time $(\tau)$ is $35 \pm 23$ ps..

\begin{tabular}{|c|c|c|c|}
\hline Wavelength $\left(\mathrm{cm}^{-1}\right)$ & Amplitude ( $\triangle O D)$ & $\tau(p s)$ & Offset ( $\triangle O D)$ \\
\hline 2038 & -0.0069 & 19 & 0.00072 \\
\hline 2025 & 0.0021 & 35 & 0.00041 \\
\hline 1990 & -0.0031 & 17 & -0.00008 \\
\hline 1979 & -0.0134 & 16 & -0.00784 \\
\hline 1964 & 0.0026 & 49 & 0.00015 \\
\hline 1943 & 0.0037 & 37 & -0.00011 \\
\hline 1899 & 0.0015 & 80 & 0.00111 \\
\hline
\end{tabular}



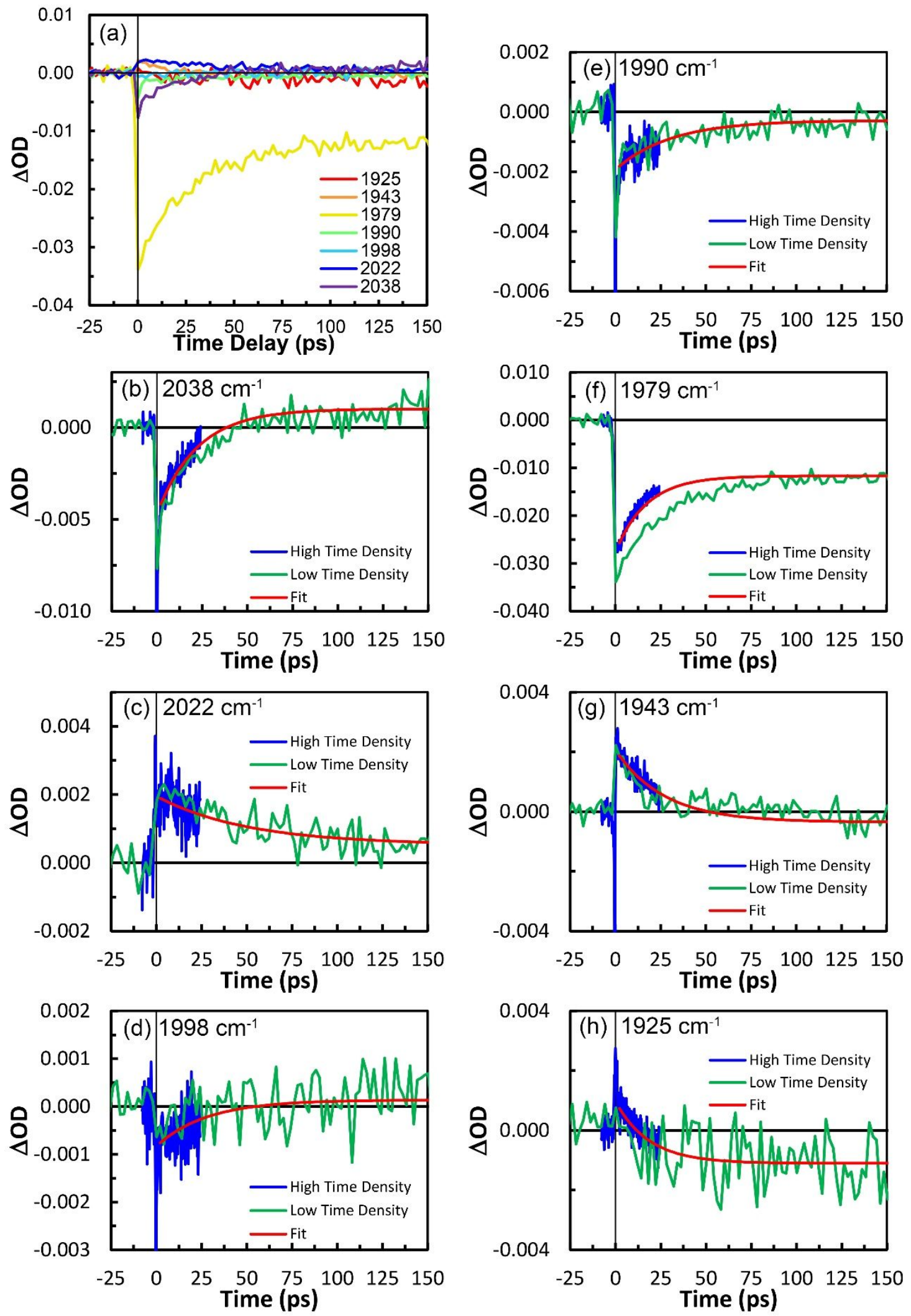

Figure S1. Exponential decay fits for seven wavelengths for 1 using $400 \mathrm{~nm}$ excitation. 


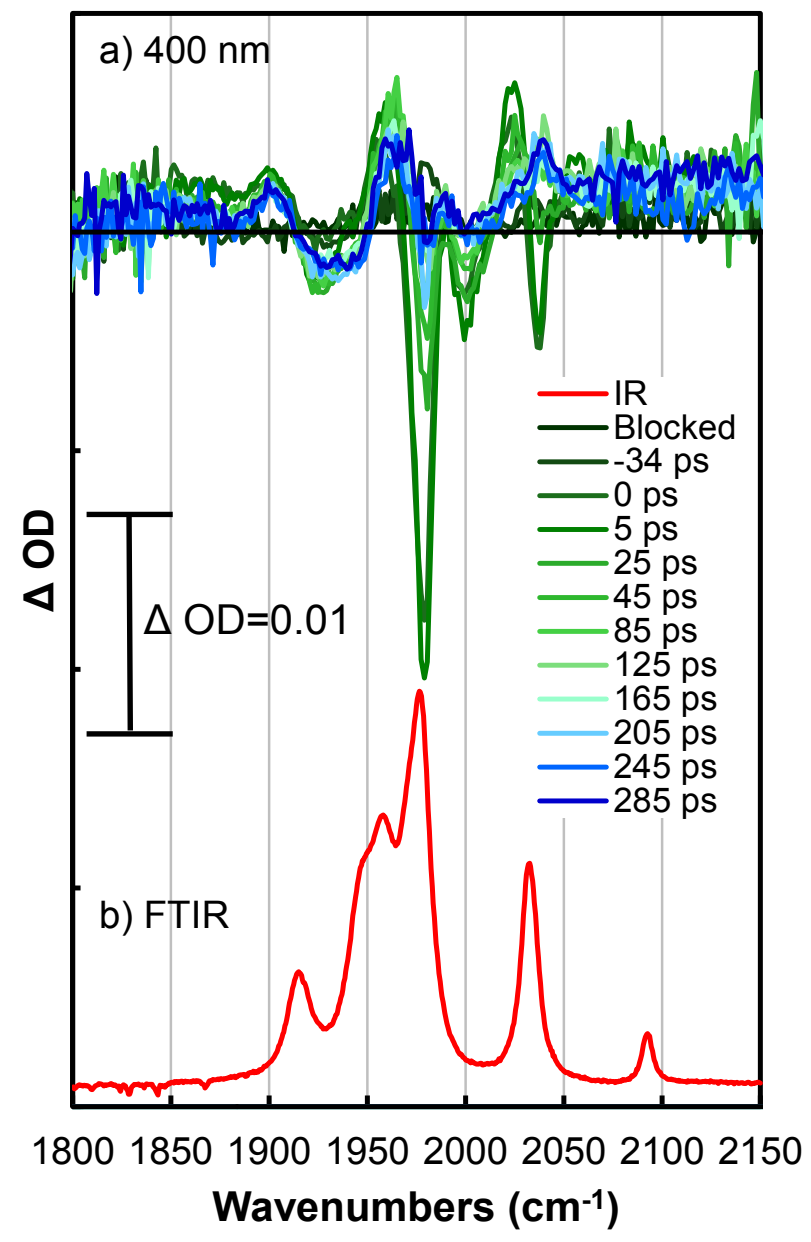

Figure S2. (a) Time-resolved $400 \mathrm{~nm}$ excitation, IR probe spectra for $\mathbf{2}$ in $\mathrm{MeCN}(298 \mathrm{~K})$ at indicated time delays after excitation and (b) ground-state FTIR spectrum of $\mathbf{2}$ in room temperature $\mathrm{MeCN}$ solution. 

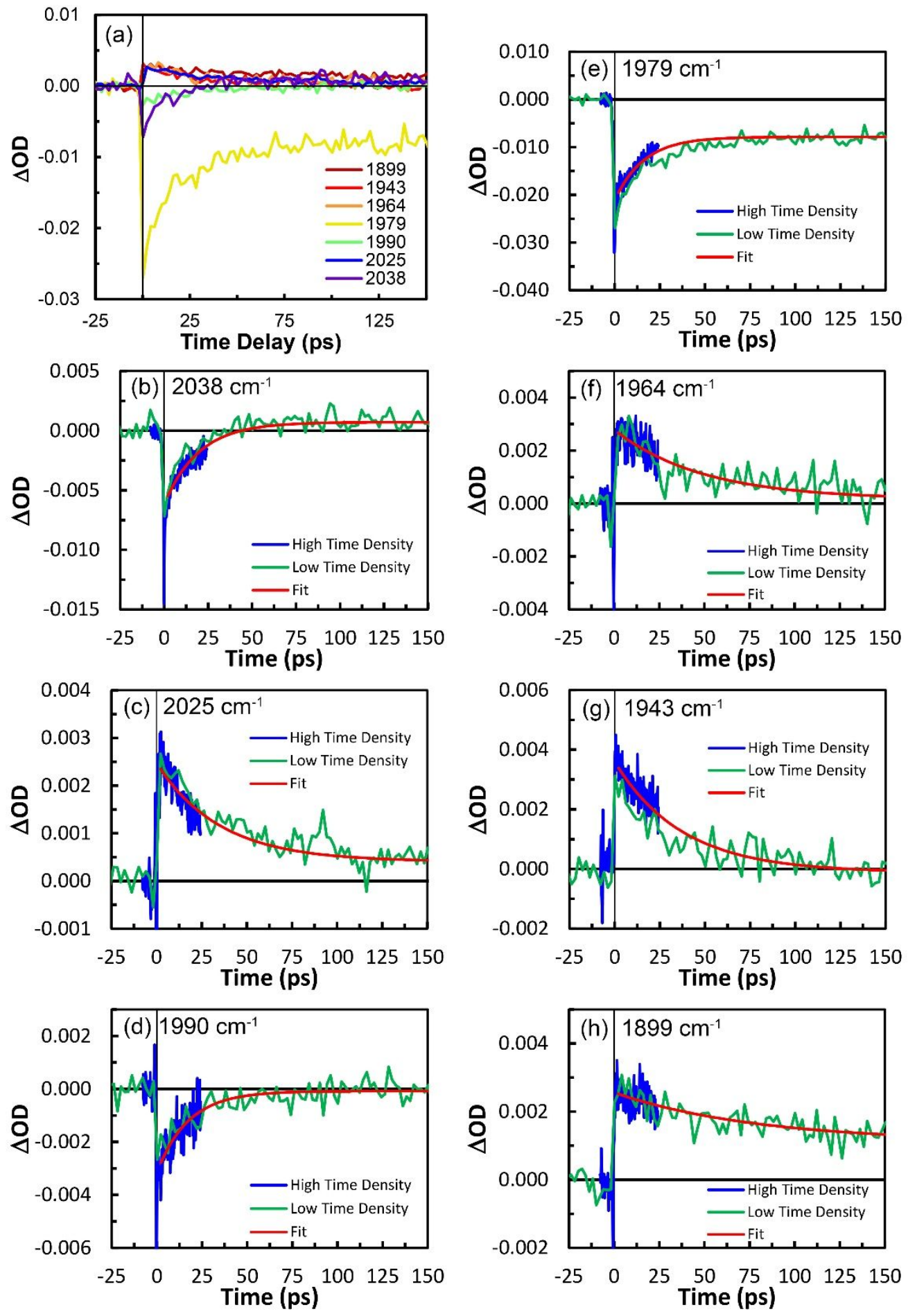

Figure S3. Time-dependent scans for 2 in MeCN (298 K) using $400 \mathrm{~nm}$ pump at (a) all selected wavenumbers, $(b-h)$ selected wavelengths. The red traces are a single-exponential decay with constant offset fits. 


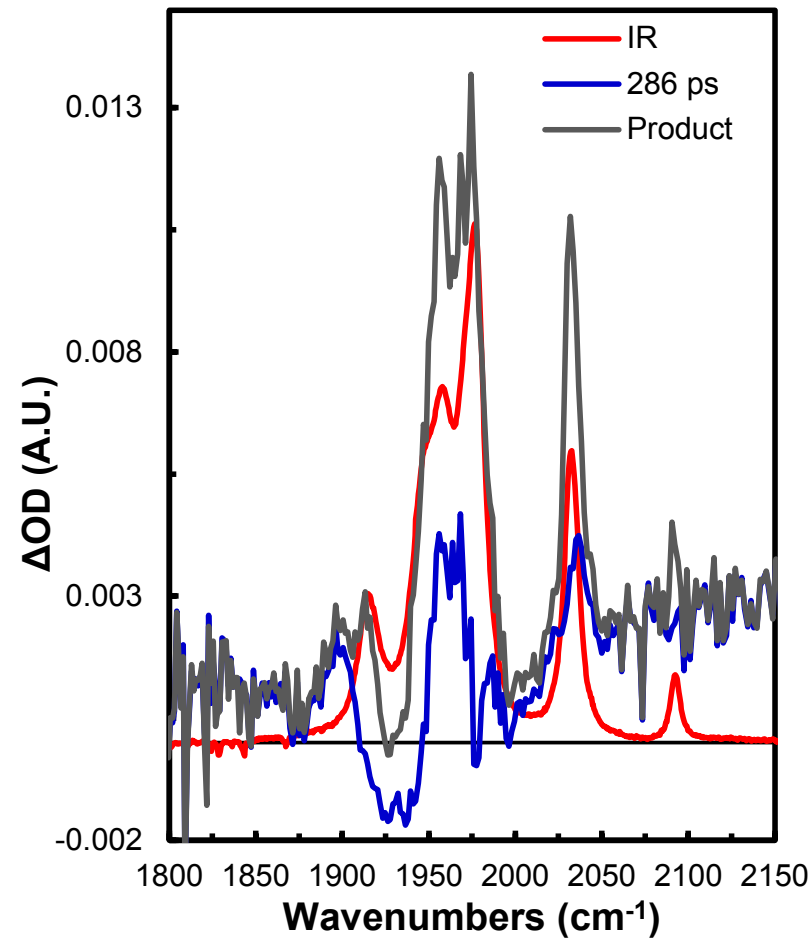

Figure S4. Long time-delay product spectrum extrapolated from the 286 ps longest delay and the FTIR spectrum for $\mathbf{2}$ in $\mathrm{MeCN}$. 

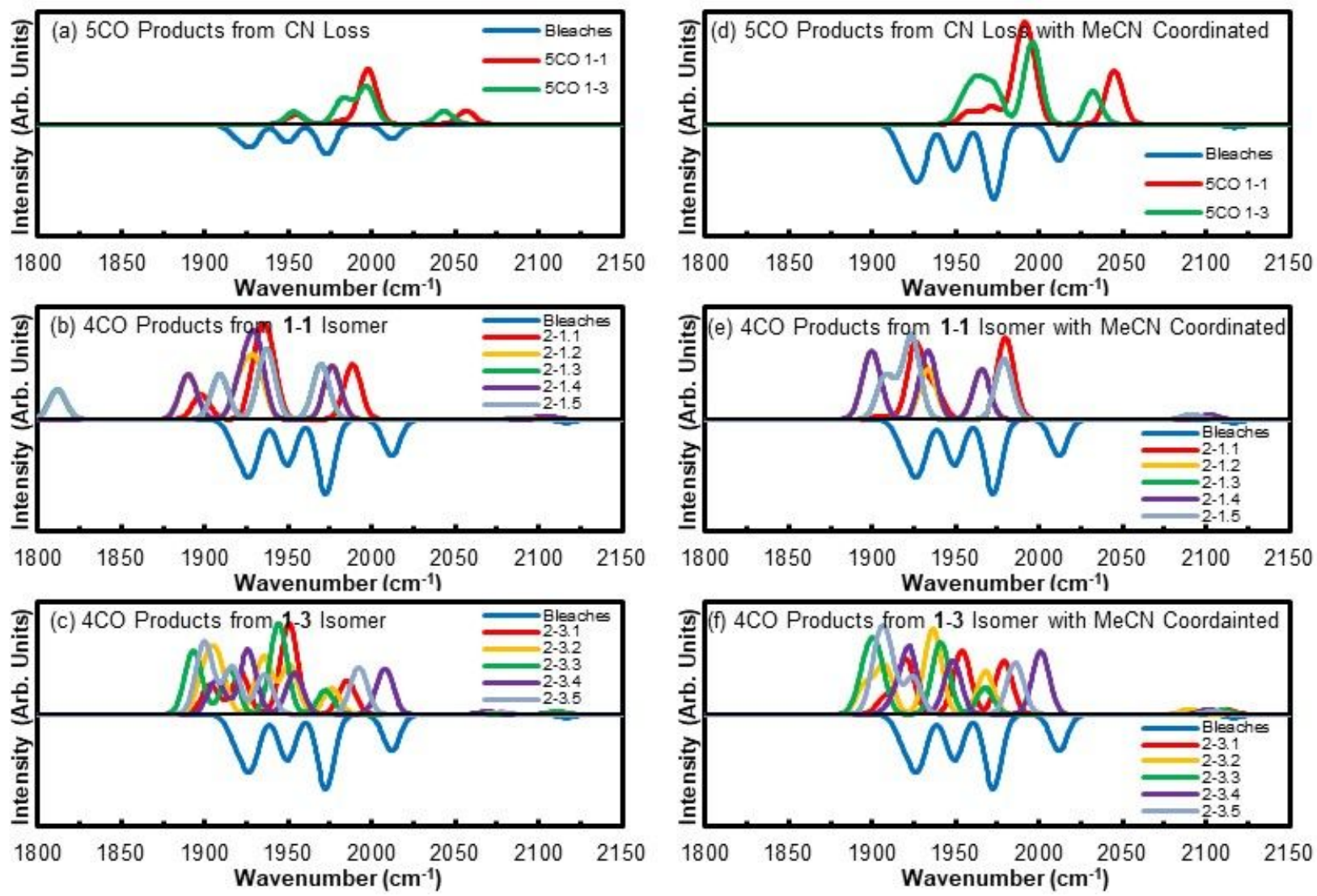

Figure S5. Calculated DFT spectra for all $\mathrm{CN}$ and CO-loss photoproduct isomers of 2: (a) CNloss isomers and $(\mathrm{b}, \mathrm{c}) \mathrm{CO}$-loss isomers originating from all possible ground state isomeric structures. The feature at $1810 \mathrm{~cm}^{-1}$ in (b) corresponds to a bridge-bonded $\mathrm{Fe}-\mathrm{CO}-\mathrm{Fe}$ product. (d-f) are the same as (a-c) but with a coordinated acetonitrile molecule in the vacant position. 


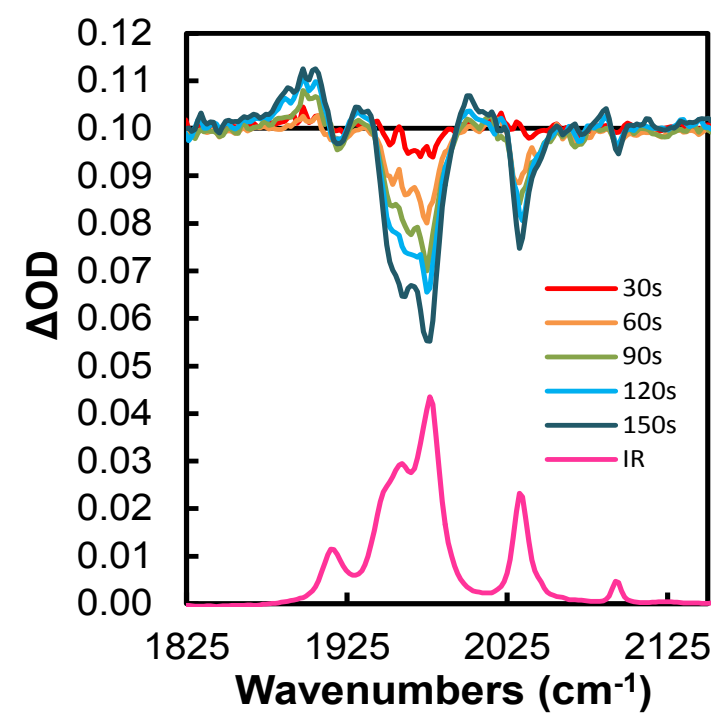

Figure S6. Long time UV-FTIR difference spectra for $\mathbf{2}$ at the indicated total UV-lamp exposure times. 


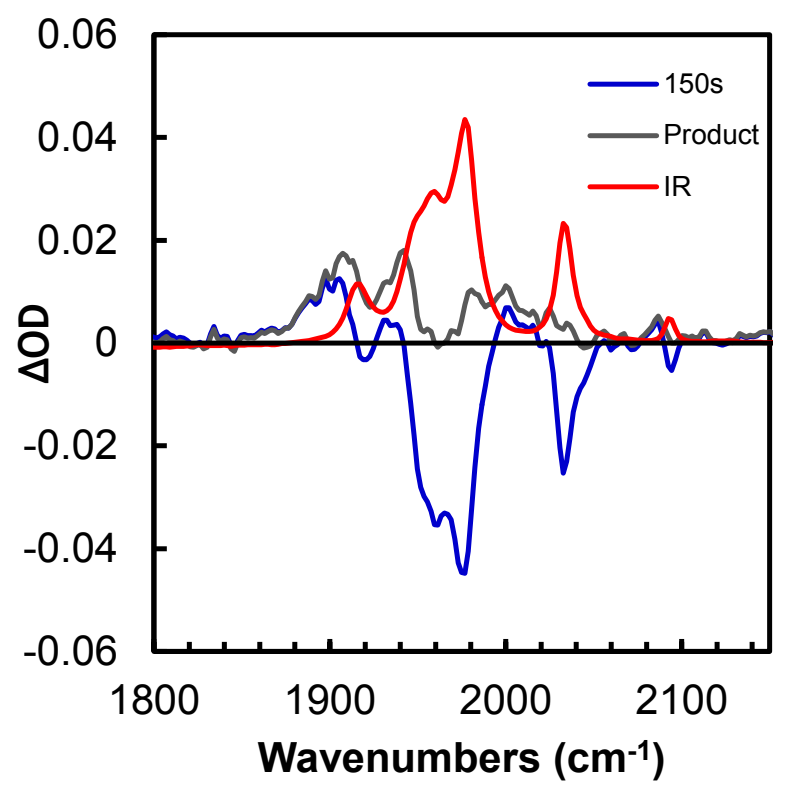

Figure S7. Long time product spectrum extrapolated from $150 \mathrm{~s} \mathrm{UV} \mathrm{exposure} \mathrm{and} \mathrm{FTIR}$ spectrum for 2. 
(a)

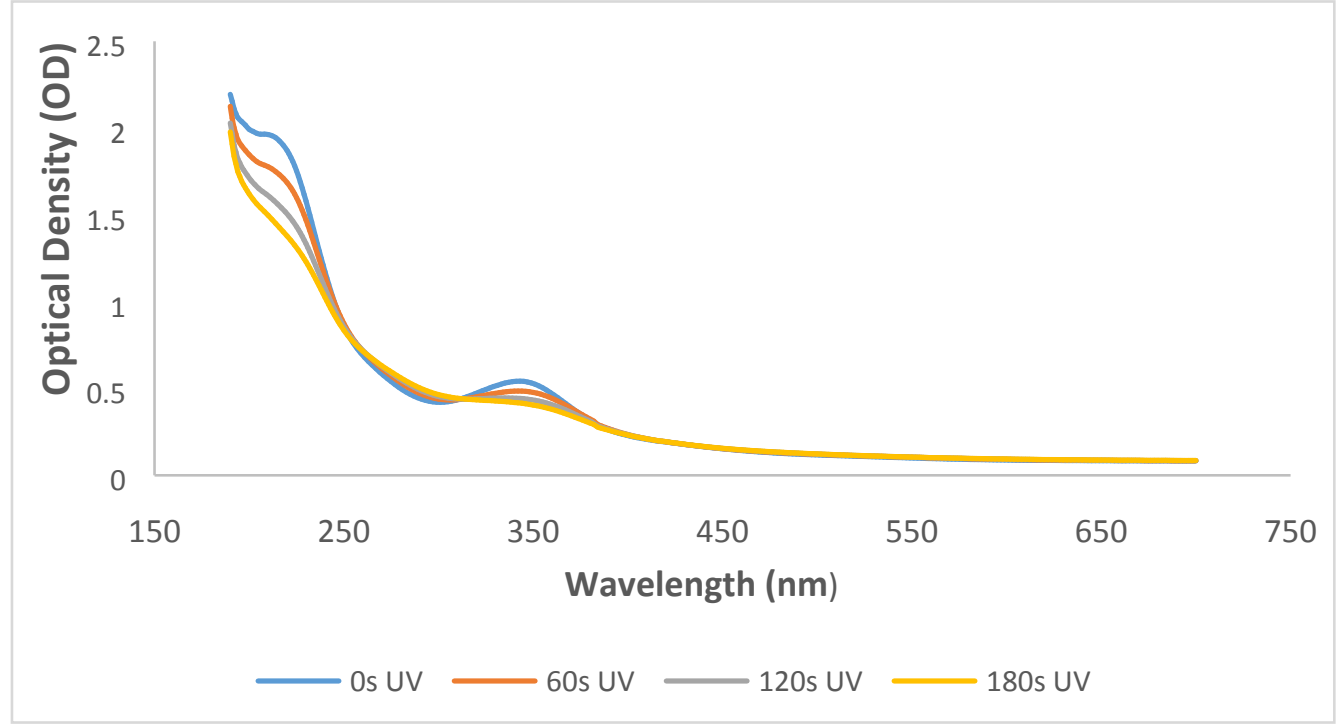

(b)

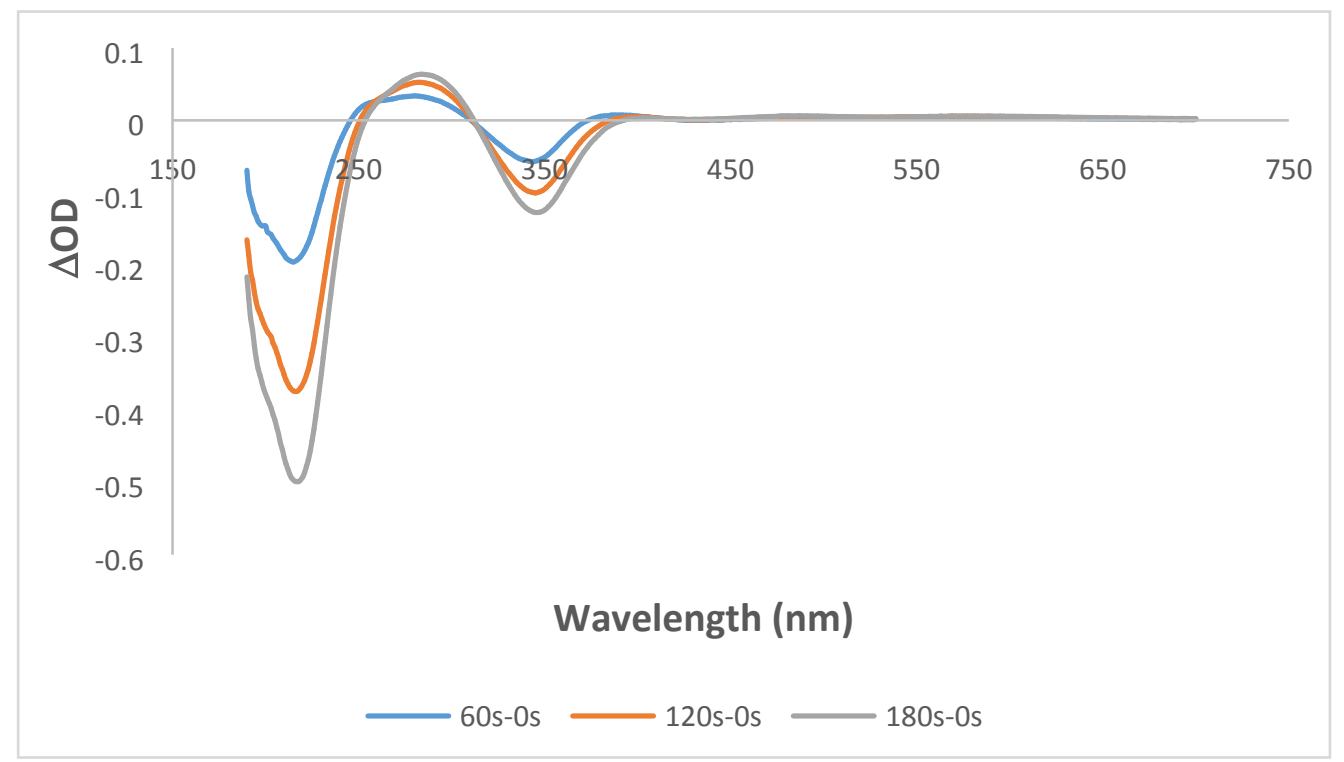

Figure S8. Unexposed and long-time delayed UV-Vis spectra after UV (Hg lamp) excitation for dilute $\mathbf{2}$ in room temperature acetonitrile exhibiting in (a) isosbestic points indicative of stable product formation and (b) Optical Density difference spectra obtained from (a) showing bleach of ground state absorptions and generation of new absorption near $290 \mathrm{~nm}$. DFT molecular orbital calculations (BP86/TZVP) for ground state 2 and coordinated with MeCN solvent after CO-ejection suggest electronic transitions occur in the $300 \mathrm{~nm}$ to $540 \mathrm{~nm}$ range with ca. $30 \mathrm{~nm}$ red-shift for product bands, but more detailed work is required to determine and assign product species. 